\title{
IMPACTOS Y RIESGOS EN LA VIOLENCIA DE GÉNERO CONTRA LAS MUJERES EN CONFINAMIENTO POR LA COVID 19: ¿QUÉ PASÓ EN ECUADOR?
}

IMPACTS AND RISKS IN GENDER-BASED VIOLENCE AGAINST WOMEN IN CONFINEMENT DUE TO COVID-19: ¿WHAT HAPPENED IN ECUADOR?

\section{Ana Veloz Avendaño'}

\section{Kathy Llori Otero²}

\section{Jorge Geovanny Tocto Lobato ${ }^{3}$}

Licença CC BY:

Artigo distribuído sob os termos Creative Commons, permite uso e distribuição irrestrita em qualquer meio desde que o autor credite a fonte original.

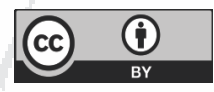

Resumo: El presente artículo tiene como objetivo demostrar los impactos y riesgos en la violencia de género contra las mujeres en el confinamiento por el coronavirus y las políticas públicas adoptadas por los gobiernos, especialmente el del Ecuador. Es un estudio descriptivo que se sustenta en los informes de las organizaciones internacionales de protección a las mujeres y de los diferentes medios de radiodifusión digital. Como resultado se identificó, que en las primeras semanas del confinamiento las mujeres no pudieron acudir a denunciar debido al confinamiento y la restricción de movilización, a diferencia que actualmente se conoce que existe un número incalculable de mujeres víctimas de violencia ejercida por la pareja durante el confinamiento, y que las políticas adoptadas no han disminuido este tipo de violencia; al contrario, se va incrementando.

Palavras-chave: violencia, género, pandemia coronavirus, confinamiento, políticas públicas.

1 Doctoranda Universidad Católica Andrés Bello UCAB, Caracas, Venezuela. Doctora y Abogada en Derecho Universidad Particular de Loja UTPL, Loja, Ecuador Master Derecho Civil UNIANDES. Docente pregrado posgrado Universidad Nacional de Chimborazo UNACH- Universidad Estatal de Bolívar UEB. Investigadora de Género y Derechos Humanos. E-mail: anavelapci@hotmail.com

2 Doctora en Ciencias Odontológicas Universidad de Zulia-Venezuela. Magister en Administración mención Gerencia de Recursos Humanos por competencias, Universidad Nacional de Chimborazo UNACH. Diplomado Superior en Políticas y Gestión de Sistemas de Salud, universidad San Francisco USFQ. kllory@unach.edu.ec.

3 Licenciado en Mención Educación Física universidad Estatal de Bolívar. Mg. En Cultura Física y Deportología. Universidad Regional Autónoma de los Andes, UNIANDES. Sport management, Universidad de Florencia ISEF. Docente de la Escuela Superior Politécnica de Chimborazo ESPOCH y Universidad Nacional de Chimborazo UNACH. Email: jtocto@espoch.edu.ec. 
Abstract: This article aims to demonstrate the impacts and risks of gender-based violence against women in confinement due to the coronavirus and the public policies adopted by governments, especially that of Ecuador. It is a descriptive study that is based on the reports of international organizations for the protection of women and the different digital broadcasting media. As a result, it was identified that in the first weeks of confinement, women could not come to report due to confinement and the restriction of movement, unlike the fact that it is currently known that there is an incalculable number of women who are victims of violence exerted by their partner during the period. confinement, and that the policies adopted have not reduced this type of violence; on the contrary, it is increasing.

Keywords: violence, gender, coronavirus pandemic, confinement, public policies.

\section{INTRODUCCIÓN}

La violencia contra la mujer es una de las manifestaciones que tienden aumentar en cualquier tipo de emergencia como las pandemias. A pesar, que durante la pandemia por COVID-19 no existe cifras sistematizadas que permitan conocer esta realidad, los informes de diferentes países del mundo, entre ellos China, el Reino Unido, Estados Unidos de América, indican que aumentó considerablemente los casos de violencia doméstica agravada por las medidas de confinamiento, distanciamiento físico y las restricciones de movilidad que incrementaron el encierro de las mujeres.

A diferencia, informes de otros países apuntan a que ha disminuido el número de víctimas, lo que hace reflexionar que las medidas de confinamiento y el miedo a infectarse en los centros de atención sanitaria, no les permitió denunciar y tampoco acudir a recibir atención médica y otros servicios como las líneas telefónicas de ayuda, los centros para la atención de crisis, los refugios, la asistencia jurídica y los servicios de protección.

Frente a la situación de la violencia de género contra las mujeres, los gobiernos y los encargados de las políticas no contaban con los planes de preparación y respuesta a la pandemia de COVID-19.

Esta investigación pretende crear una memoria de la violencia de género perpetrada contra las mujeres durante el confinamiento por la pandemia por COVID-19, desde los hallazgos recopilados de los medios digitales. Planteando las siguientes inquietudes:

¿Cuáles son los impactos y riesgos en las mujeres víctimas de violencia por el confinamiento debido a la pandemia por COVID-19?

¿Cuál fue el impacto de las políticas públicas adoptadas por los Estados frente a la violencia contra las mujeres durante el confinamiento por COVID- 19? 


\section{LA VIOLENCIA DE GÉNERO CONTRA LAS MUJERES}

La violencia de género, ha sido y sigue siendo una de las manifestaciones más claras de la desigualdad, subordinación, y de relaciones de poder del género masculino sobre el femenino. Como señala Maqueada: "(...) se trata de una violencia que se dirige sobre las mujeres por el hecho mismo de serlo" ${ }^{4}$. La Plataforma de Acción de Beijing reconoce que la violencia contra las mujeres, es ejercida por el dominio que ejercen los hombres sobre ellas, debido a los imaginarios de poder, discriminación y subordinación, lo que atentata su pleno desarrollo ${ }^{5}$. El mismo organismo, reconoce que la violencia contra las mujeres se deriva de aspectos culturales, incluidas las prácticas tradicionales o consuetudinarias y los actos de extremismo relacionados con la raza, el sexo, el idioma o la religión que dañan a las mujeres y les otorgan un estatus inferior en familia, lugar de trabajo, comunidad y sociedad 6 . Desde el espacio de análisis de los derechos humanos, la violencia de género, significa la restricción a la libertad, a la dignidad y la libre movilidad de las personas, es una violación directa de su integridad? ${ }^{7}$ Galtung definió que la violencia es estructural, que se produce como consecuencia de los elementos estructurales de la cultura y la organización de una sociedad determinada ${ }^{8}$.

\section{FACTORES DE RIESGO DE LA VIOLENCIA DE GÉNERO CONTRA LAS MU-} JERES

Los factores de riesgo al que se someten debido a la VG, como una cuestión de salud pública, es una de las causas más importantes de morbilidad y mortalidad de las mujeres durante sus años reproductivos $^{9}$. Además de las lesiones físicas, las mujeres violentadas llegan a sufrir estrés crónico y otras enfermedades como hipertensión, diabetes y asma; además de otros padecimientos tales como trastornos sexuales, depresión, fobias y miedos ${ }^{10}$.

Según, Estefanía Molina Giraldo, considera que son la cultura patriarcal, la construcción de roles en función del género, la exclusión social. Entre los demográficos señala la pobreza, el consumo de alcohol, el consumo de sustancias alucinógenas, el desempleo o las dificultades laborales y el bajo

4 MAQUEDA, María Luisa. La violencia de género: Entre el concepto jurídico y realidad social, Revista Electrónica de Ciencia Penal y Criminología 2006, p.4.

5 Resolución 48/104 de la Asamblea General de las Naciones Unidas. Cfr. IV Conferencia Internacional de Beijing de. Revista del Ministerio de Trabajo y Asuntos Sociales. Número 42. Pág. 231. Cfr. Convención Interamericana para prevenir, punir y erradicar la violencia contra la mujer, de 1994.

$6 \quad$ NACIONES UNIDAS. IV Informe de la Cuarta Conferencia Mundial sobre la Mujer, 1995, p.52.

7 BUNCH, Charlotte, HINOJOSA, Claudia y REILLY, Niamh. Los derechos de las mujeres son derechos humanos, crónica de una movilización mundial. Edamex S.A. México 2000.

8 GALTUNG J. Violence, peace, and peace research, Journal of Peace Research, vol., 6, pp. 167-191,1969 en GARCÍA, Dora. En busca de una cultura de Paz frente a la violencia: El caso de la Trata de personas. Frontera Norte, vol. 26, núm. 51. 2014, págs. 181-192.

9 SAGOT, Montserrat. The Critical Path of Women Affected by Family Violence in Latin America Case Studies From 10 Countries. Violence Against Women, 2005, pgs. 1292-1318.

10 LARRAín, S. Los orígenes y el control de la violencia doméstica en contra de la mujer, 1993, en GÓMEZ, Elsa, Género, Mujer y Salud en las Américas, Washington D.C. Organización Panamericana de la Salud,1993. págs. $202-212$. 
nivel de educación ${ }^{11}$. Entre los motivos pasionales, los celos, los conflictos de pareja y la influencia de las emociones, pero los más predominantes dice que son la aceptación de la violencia y la baja autoestima $^{12}$. La OMS destaca los antecedentes de violencia; la discordia e insatisfacción marital; las dificultades de comunicación entre los miembros de la pareja; la conducta dominadora masculina hacia su pareja ${ }^{13}$.

Otro factor de riesgo es el confinamiento, que crea las condiciones para que la VG se incremente debido al encierro obligatorio en el hogar, aisla e impide el pedir ayuda o denunciar lo que incrementa la impunidad del agresor y le facilita realizar cualquier forma de violencia ante una mínima incitación. orden, coarta las libertades y ejerce violencia sobre la mujer ${ }^{14}$.

\section{ALARMA POR EL INCREMENTO DE LA VIOLENCIA DE GÉNERO EN EL CONFINAMIENTO POR COVID-19}

Actualmente el mundo está condenado a la propagación mundial de una nueva enfermedad por coronavirus COVID-19, por la cantidad de personas infectadas y muertas debido a esta infección en la mayoría de países en todo el mundo, la Organización Mundial de la Salud (OMS) concluyó que la COVID-19 es una "pandemia"15. En estas circunstancias, los gobiernos de los países han tomado una serie de medidas para evitar una mayor transmisión, desde recomendar a la población a intensificar las medidas de salud hasta limitar el movimiento de personas, obligando a estar confinadas en sus hogares para evitar la propagación ${ }^{16}$.

Al decretar estas medidas, los gobiernos no tuvieron presente, ni se imaginaron el alto impacto y/o riesgos al que están expuestos los distintos sectores vulnerables de la sociedad, particularmente las "mujeres" que están confinadas obligatoriamente en sus hogares junto a sus agresores, lo que ha agudizado en las primeras semanas del confinamiento los sucesos de la violencia latente (escondida), traspasado a las fases de explosión durante la pandemia, como pasó en China y Corea, posteriormente en Italia, España y Francia, y ahora lamentablemente, en América Latina y el Caribe, según la ONU ${ }^{17}$.

11 MOLINA, Estefanía. Factores de riesgo y consecuencias de la violencia de género en Colombia, Tempus Psicológico, 2019, Pgs. 15-36.

12 MOLINA, Estefanía. Factores de riesgo y consecuencias de la violencia de género en Colombia, 2019, Pgs. $15-36$

13 OMS (Organización Mundial de la Salud). Obtenido de Violencia contra la Mujer, 2017, consultado en: https://www. who.int/es/news-room/fact-sheets/detail/violence-against-women.

14 LORENTE, Miguel. Violencia de género en tiempos de pandemia y confinamiento. Revista española de medicina legal, 2020, Pgs. 139-145.

15 OMS (Organización Mundial de la Salud). La OMS caracteriza a COVID-19 como una pandemia. 2020, consultado en: https://www.paho.org/es/noticias/11-3-2020-oms-caracteriza-covid-19-como-pandemia.

16 CEPAL (Comisión Económica para América Latina y el Caribe). Medidas y acciones impulsadas por los Gobiernos de América Latina y el Caribe frente al COVID-19 en áreas clave para la autonomía de las mujeres y la igualdad de género. 2021, consultado en: https://www.cepal.org/sites/default/files/events/files/220222_ documento_mapeo_medidas_covid-19_rev_dag_002.pdf

17 OEA/CIM. (Organización de los Estados Americanos/ Comisión Interamericana de Mujeres). Covid-19 en la vida de las mujeres. Razones para reconocer los impactos diferenciados. 2020. Consultado en: https://www.oas. org/es/cim/docs/ArgumentarioCOVID19-ES.pdf 
El confinamiento por la pandemia de la COVID-19, ha llevado a que varias organizaciones de protección de derechos de las mujeres se pronuncien, como Nubravka Simonovic relatora especial de Naciones Unidas, señala que, para demasiadas mujeres y niños, el hogar puede ser un lugar de miedo y abuso ${ }^{18}$. Así también, en una de sus últimas intervenciones el Secretario General de Naciones Unidas, António Guterres, se ha referido que la desigualdad de género se ha incrementado más aún en esta pandemia, lo que lleva a que se realicen un esfuerzo especial para cumplir el ODS 5, relativo a la igualdad de género ${ }^{19}$.

Otras organizaciones tales como ONU Mujeres para las Américas y el Caribe y la Cruz Roja Española, advirtieron que el confinamiento aumentaría la sensación de control por parte del maltratador y de dependencia por parte de la víctima ${ }^{20}$. De igual manera, las personas sobrevivientes de violencia pueden enfrentar obstáculos adicionales para huir de situaciones violentas o para acceder a servicios de atención, protección y/o servicios esenciales que pueden salvar sus vidas ${ }^{21}$.

Miguel Lorente, exdelegado del Gobierno contra la VG de España, comenta: "los agresores perciben impunidad y seguridad, porque el encierro dificulta salir de la relación o interponer una denuncia"22. La jurista Lucía Avilés, portavoz de la Asociación de Mujeres Juezas de España (AMJE), reitera que los confinamientos traen consigo la obligación de convivir con el agresor, quien ejerce el control hasta de las comunicaciones $y$, al tener coartada la libertad de movimiento, muchas mujeres sienten reducidas sus posibilidades de pedir ayuda. Además, expresó preocupaciones particulares sobre las mujeres con mayor riesgo de violencia doméstica, como las mujeres con discapacidad, las mujeres migrantes indocumentadas y las víctimas de la trata ${ }^{23}$.

Lo que ratifica la jurista croata, especialista en políticas de igualdad entre hombres y mujeres y Derechos Humanos, Dubravka Šimonović: Para empeorar las cosas, las restricciones de movimiento, las restricciones financieras y la incertidumbre generalizada envalentonan a los perpetradores y lesproporcionan poder y controles adicionales. Otros aspectos son la sobrecarga de trabajo enel hogar, aquellas que se encuentran embarazadas temen por su salud a la hora de asistir a

18 GARCÍA, Noemi. Coronavirus y violencia de género: un binomio peligroso. Ayuda en Acción, (20 de abril de 2020). Consultado en: https://ayudaenaccion.org/ong/blog/mujer/coronavirus-violencia-genero/.

19 CEPAL (Comisión Económica para América Latina y el Caribe). Construir un futuro mejor: acciones para fortalecer la Agenda 2030 para el Desarrollo Sostenible (LC/FDS.4/3/Rev.1). Santiago, 2021. Consultado en: https://repositorio.cepal.org/bitstream/handle/11362/46682/6/S2100125_es.pdf

2020 Minutos. Cruz roja advierte que el confinamiento puede aumentar las agressiones a las victimas de violencia de género. Google, (2 de abril de 2020). Obtenido de: https://www.20minutos.es/noticia/4214837/0/ cruz-roja-advierte-que-el-confinamiento-puede-aumentar-agresiones-a-las-victimas-de-violencia-de-genero/.

$21 \mathrm{BBC}, \mathrm{R}$. N. Coronavirus: la preocupación por las víctimas de violencia de género que tienen que convivir en cuarentena con su agresor (y dónde buscar ayuda). (24 de marzo de 2020). BBC, https://www.bbc.com/ mundo/noticias-52009140.

22 EL PAíS. Encerradas con el maltratador, (20 de marzo de 2020). https://elpais.com/sociedad/2020-03-20/ encerradas-con-su-maltratador.html. PREGUNTAR QUE PÁGINA SE PONE MEDIO DIGITAL

23 MOLINA, Violeta. La denuncia del negocio de la violencia de género con el COVID 19, pero nada para los varones confinados con sus maltratadoras, 2020. Recuperado de: https://www.efe.com/efe/espana/sociedad/ violencia-machista-prohibido-dar-la-espalda-durante-pandemia/10004-4198583 


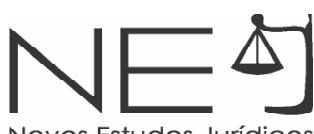

controles,o aquellas mujeres trabajadoras sexuales que son más explotadas ${ }^{24}$. Entre estas y otras, también fueron las preocupaciones de Rosa Muñoz Lima, psicóloga, comunicadora e investigadora social, quien advirtió que la pandemia acarrearía "situaciones de vulnerabilidad, de inseguridad, de exposición, de pérdida de asideros materiales y espirituales, incrementa la respuesta violenta y las mayores víctimas serían las mujeres y los menores, como ha pasado en otras pandemias o desastres ${ }^{25}$. Además, Dubravka Šimonović de ONU Mujeres, presagió que habría un repunte de los asesinatos machistas, es decir femicidios ${ }^{26}$.

Todas las advertencias de las organizaciones internacionales sobre el incremento y las consecuencias de la violencia de género no fueron simples presagios, la VG, según el jefe de la oficina del organismo de la ONU para Europa, Hans Kluge, alertó que la violencia contra las mujeres aumento en un $60 \%$, también que se quintuplicaron las llamadas a los teléfonos de ayuda, y advierte "si las medidas de confinamiento se extienden por seis meses más, habrá 31 millones de nuevos casos de violencia doméstica alrededor del mundo" (...), esto es inaceptable, expresó27.

Una tras otra historia de las miles de mujeres a nivel de todo el mundo se hicieron visibles en los medios digitales, para recordar el caso de Karina de 28 años, quien teme que su agresor le encuentre y termine lo que empezó la última noche que la golpeó y la violó, durante el encierro por el coronavirus la vida de ella fue un infierno, no solo por la cuarentena sino porque cuando su maltratador salía, la dejaba encerrada con sus hijos. Dice Karina, "comenzó a estar más alterado cuando dejó de haber dinero en casa, los niños estaban conmigo y vivían toda esa violencia", consiguió huir gracias a que la puerta se quedó abierta una mañana ${ }^{28}$.

El circulo de la violencia se agudiza durante la pandemia y no sabemos cuántas mujeres han muerto en manos de sus agresores, no existen cifras oficiales, pero están muertas. Como aquel suceso que causó alarma, porque fue asesinada delante de sus dos hijos menores en Almassora (Castellón-España); de este caso, no había denuncias previas de violencia ${ }^{29}$;

24 NOTICIAS ONU, EI COVID-19 golpea tres veces a las mujeres: por la salud, por la violencia doméstica y por cuidar de los otros, 2020. Recuperado de: https://news.un.org/es/story/2020/03/1471872

25 MUÑOZ, Rosa. América Latina lucha contra la violencia de género en tiempos del coronavirus. DW Made For Minda , (03 de 31 de 2020). Recuperado de : https://www.dw.com/es/am\%C3\%A9rica-latina-lucha-contra-laviolencia-de-g\%C3\%A9nero-en-tiempos-del-coronavirus/a-52971832.

26 ONU (Organización de las Naciones Unidas). Las trabajadoras domésticas en jaque por el coronavirus, (20 de junio de 2020). Noticias ONU.

27 KLUGE, Hans. La OMS alertó que la violencia contra las mujeres aumento un $60 \%$ durante las cuarentenas (08 de mayo de 2020), infobae, págs. s/n. recuperado de:https://www.infobae.com/america/mundo/2020/05/07/ la-oms-alerto-que-la-violencia-contra-las-mujeres-se-incremento-un-600-por-las-cuarentenas/.

28 BARRAGAN, Almudena, Y RODRÍGUEZ, Darinka. Las llamadas por violencia de género en México aumentan $60 \%$ durante la cuarentena, (03 de 04 de 2020), EL PAÍS. https://verne.elpais.com/verne/2020/04/02/ mexico/1585780887 471083.html.

29 GARCÍA DE BLAS, Elsa. Encerradas con su matratador, (20 de marzo de 2020), EL PAÍS, https://elpais.com/ sociedad/2020-03-20/encerradas-con-su-maltratador.html. 
Otro caso que desencadeno en femicidio por estrangulamiento, en Italia (Messina), doctora de 27 años de edad, quien combatía la COVID-19, asesinada por su novio tras la desesperación que su pareja estaba contagiada y le había infectado de COVID-19; realizadas las pruebas médicas, le informaron que ninguno de los dos era portador del virus ${ }^{30}$.

Además, en algunos refugios de mujeres se han duplicado las solicitudes de asilo por violencia, su capacidad al máximo y sin presupuesto estatal que garantice mantenerse abiertos los próximos meses $^{31}$. El riesgo se agrava cuando hay menos apoyo de la comunidad; menos intervenciones policiales y menos acceso a la justicia ya que muchos tribunales están cerrados"32.

De acuerdo al diario chino Global Times, luego de que las cifras de contagio por coronavirus disminuyeron en ese país, el número de divorcios y denuncias por casos de violencia doméstica en algunas ciudades alcanzaron un pico como consecuencia del aislamiento por este brote. Así como un aumento sin precedente de las solicitudes de divorcio en las oficinas de registro matrimonial de Xi'an, capital de la provincia de Shaanxi, en el noroccidente de China ${ }^{33}$.

Otro factor que influye en la VG, es el aumento de consumo de alcohol detectado durante la cuarentena, los motivos por los que aluden beber es la ansiedad, estrés, aburrimiento y depresión debido al COVOD -19, según un estudio realizado por el Ministerio de Salud de Chile ${ }^{34}$.

\section{POLÍTICAS PÚBLICAS PARA EVITAR LA VIOLENCIA DE GÉNERO}

Ante esta realidad latente de la violencia de género contra las mujeres, la Declaración sobre la eliminación de la violencia contra la mujer y la Recomendación General No. 19 de la CEDAW, adoptaron el concepto de obligación de debida diligencia de los Estados ${ }^{35}$. Según esta Declaración, los Estados tienen el deber de adoptar medidas positivas para prevenir la violencia contra las mujeres y protegerlas, sancionar a quienes cometen actos de violencia y compensar a las víctimas de dichos actos.

30 DIARIO INTERNACIONAL, Aumentan reportes de violencia de género durante confinamiento en España, (04 de abril de 2020), Recuperado de : https://www.adn40.mx/noticia/internacional/notas/2020-04-03-14-59/italiaprotesta-contra-feminicidio-de-doctora-que-combatia-el-covid-19.

31 BARRAGAN, Almudena y RODRÍGUEZ, Darinka. (03 de 04 de 2020). Las llamadas por violencia de género en México aumentan $60 \%$ durante la cuarentena. EL PAíS, pág. https://verne.elpais.com/verne/2020/04/02/ mexico/1585780887_471083.html.

32 ONU (Organización de las Naciones Unidas), Las trabajadoras dompesticas en jaque por el coronavirus, (20 de junio de 2020), Noticias ONU.

33 KOHAN, M., Indignación en el Ministerio de Igualdad ante el afán de Interior por minimizar la violencia machista en cuarentena. Diario Público de Madrid, 31 de marzo de 2020, Recuperado de: https://www.publico. es/sociedad/violencia-genero-coronavirus-indignacion-ministerio-igualdad-afan-interior-minimizar-violenciamachista-cuarentena.html.

34 SENDA. Segunda encuesta online efectos del COVID-19 en el uso de alcohol y otras drogas en Chile 2021. Recuperado de: https://www.senda.gob.cl/wp-content/uploads/2021/06/Segunda-Encuesta-SENDA.pdf

$35 \mathrm{ACNUDH}$. (Alto Comisionado de las Naciones Unidas para los Derechos Humanos). Declaración sobre la eliminación de la violencia contra la mujer. 2000. Recuperado de: http://www.oas.org/DIL/ESP/1993. Declaracion_sobre_la_eliminacion_de_la_violencia_contra_la_mujer.pdf. 
Frente al aumento en los casos de violencia doméstica, abuso sexual en niñas, casos de femicidios, los Estados han creado medidas para abordar la violencia contra las mujeres en sus diferentes manifestaciones, en cada uno de los países los gobiernos han aprobado protocolos, instructivos, guías, manuales y otros, en los que contenían mecanismos de prevención de la VG, ante el riesgo de infección las visitas domiciliarias de las redes de apoyo y servicios sociales se redujeron.

El Estado y las organizaciones de mujeres, han implementado mecanismos perceptibles como las campañas publicitarias a través de los medios televisivos y redes sociales, con mensajes en los que inducían a las mujeres a denunciar si están siendo víctimas de violencia por parte de sus parejas, como: "estamos contigo", "estamos aquí para ti", "la violencia de género la paramos unidas", las cuales brindan información y números de emergencia que buscan animar a las mujeres a reportar cualquier tipo de violencia, así como a los vecinos por la dificultad que tienen las mujeres a denunciar ${ }^{36}$.

Las tecnologías de la información juegan un papel importante para la prevención y atención a víctimas de VG, están activadas líneas telefónicas gratuitas en las entidades del Estado como la Fiscalía, policía, ECU 911, entidades que fueron reforzadas para dar una supuesta asistencia inmediata, las redes sociales como WhatsApp, con botones de pánico permiten tener la ubicación de la víctima, al igual se creado una función de geolocalización, que ubica las rutas de atención más específicas, la comunicación virtual ha permitido que las mujeres a través de códigos indiquen a sus contactos que está en situación de violencia en el hogar. En los casos que las mujeres por necesidad extrema debían salir a la farmacia, el gobierno de las islas Canarias, en España tuvo la iniciativa de crear una alerta cuando solicitan una "Mascarilla-19", mensaje que alerta al personal de las farmacéuticas, que saben que se trata de una llamada de auxilio y se pondrán en contacto con la Policía ${ }^{37}$.

Estos y cuantas medidas más deben haberse creado, pero el mismo confinamiento, el escaso acceso por parte de las mujeres a medios digitales, el miedo al agresor, las economías precarias para mantener activos los medios digitales, son factores que impiden a que la mujer pueda denunciar y quede en la impunidad el agresor.

\section{LA VIOLENCIA DE GÉNERO EN ECUADOR EN TIEMPOS DE PANDEMIA}

Así como en todo el mundo, en Ecuador la mujer es la más afectadas por la pandemia que actualmente se está atravesando, las consecuencias sociales y económicas se agudizan, la pobreza, la violencia machista, el desempleo de las mujeres rodea el 8,7\%, los salarios han disminuido, las horas de trabajo se han incrementado en 5 horas más debido al cuidado de los hijos menores y personas de la tercera edad.

36 MINDS, D. M., Confinamiento por coronavirus: la violencia doméstica y el abuso infantil podrían aumentar. 20 de 03 de 2020, recuprado de: w.com/es/confinamiento-por-coronavirus-la-violencia-doméstica-y-el-abusoinfantil-podrían-aumentar/a-52862705.

37 RADIO VALENCIA. El confinamiento aumenta el riesgo de las mujeres maltratadas. Recuperado de : https:// cadenaser.com/emisora/2020/04/01/radio_valencia/1585745709_613957.html. 
La violencia de género en todas sus formas ha aumentado por el confinamiento, pero ha disminuido el índice de denuncia. Según una encuesta realizada por Pulso Ciudadano y el Centro Ecuatoriano para la Promoción y Acción de la Mujer (CEPAM) indica que el 52,94\% de las mujeres encuestadas, sienten inseguridad de nivel 1 (en una escala de 1 a 5, en la que 5 reflejaba seguridad en el hogar, y el 81,46 \% dijo sentirse humillada o desvalorizada por su pareja durante el confinamiento, el $94,24 \%$ de las mujeres han tenido conflictos con su pareja debido a la carga laboral o desempleo, así como las tareas del hogar o crianza de los hijos, el 9,39\% de mujeres dijo haber sido víctima de violación sexual durante la cuarentena, finalmente 118 mujeres fueron asesinadas ${ }^{38}$.

Según datos de la Fiscalía General del Estado (FGE), la entidad recibió hasta el 13 de septiembre 7312 denuncias de violencia de género, 5683 han sido procesadas ${ }^{39}$. Otros datos de Primicias indica que, según fuentes de la FGE en total se registraron 19.975 casos, entre marzo y agosto (Abuso sexual 2515, Acoso Sexual 520, actos de odio 13, femicidios 15, violencia intrafamiliar 1211, violación 2213, violación incestuosa 3, violencia física contra la mujer 1888, violencia psicológica 11404, violencia sexual 93), sobre estos mismos tipos penales, en el semestre anterior (septiembre 2019 - febrero 2020) hubo 27.807 noticias del delito. Es decir, durante la emergencia hubo una reducción del -26,6\%, las tasas más altas son de las provincias pequeñas de la Amazonía ${ }^{40}$.

El Estado ecuatoriano, ante las alarmantes cifras de violencia creo un protocolo de comunicación y atención de casos de violencia de género e intrafamiliar durante la emergencia sanitaria por COVID-19, el Consejo Nacional para la igualdad de Género implemento lineamientos para su implementación en las instituciones del Estado, el Plan de respuesta humanitaria covid-19 Ecuador, la Ley Orgánica de Apoyo Humanitario (no establece ninguna medida enfocada a la prevención y atención de la violencia de género).

Los canales de denuncia de violencia de género del Estado la línea de emergencia ECU-911, para hechos flagrantes, y el número 1800DELITO opción 4, la Fiscalía General del Estado ha habilitado un formulario virtual para realizar denuncias en línea, en redes sociales grupos ciudadanos se organizan con palabras clave y mecanismos de ayuda con la etiqueta "Vendo Maquillaje", el Gobierno Autónomo provincial de Pichincha activó el protocolo de emergencia que funciona a través de llamada telefónica en la que la víctima debe responder "canasta roja"41.

38 PALOMINO F. Encuesta violencia de género contra las mujeres durante el confinamiento por el COVID-19, 2020. Recuperado de: https://www.cepam.org.ec/wp-content/uploads/2020/09/Datos_Violencia-de-Genero_ ECU2020.pdf

39 FGE. (Fiscalía General del Estado). Informe de delitos a agosto 2020, FGE, 2020.

40 PRIMICIAS, 20.000 casos de violencia de género e intrafamiliar reportados des de marzo 2020, Recuperado de: https://www.primicias.ec/noticias/sociedad/ecuador-casos-violencia-genero-emergencia/

41 ARTICULACIÓN REGIONAL FEMINISTA, "Los derechos de las mujeres de la región en épocas de COVID-19. Estado de situación y recomendaciones para promover políticas con justicia de género", Buenos Aires, mayo 2020. 
La Fiscalía para Equidad y Seguridad en Temas de Género como la Secretaría de DerechosHumanos han anunciado que durante la emergencia sanitaria continuarán funcionando cinco Casas de Acogida y dieciséis Centros de Atención Integral, que hasta el inicio de la pandemia no se había entregado el presupuesto que ha 2019 fue reducido en un $50 \%{ }^{42}$.

\section{CONSIDERACIONES FINALES}

No cabe duda que la violencia de género es estructural, viola los derechos humanos de las mujeres (salud, alimentación, la libertad, la dignidad, el derecho a una vida libre de violencia), se agudiza durante los eventos naturales, más aún en confinamiento. El coronavirus COVID-19 creo las condiciones más propicias para que se incremente, debido al aislamiento de las mujeres más de lo común, manteniéndoles en encierro obligatorio, lo que facilitó la impunidad para el agresor al no poder denunciarle, creo un contexto en el que se han dado las más crueles formas de violencia hasta consumar muchas muertes de mujeres en el mundo, lamentablemente se desconoce las cifras oficiales.

Son muchos los riesgos que se perciben en esta nueva forma de convivencia para las mujeres, siguen en riesgo la vida, la integridad física, psicológica, y sexual de las mujeres. Se agudiza la sobrecarga de labores de cuidado, aquellas mujeres que son cabeza de hogar deben poner en riesgo su salud y la de su familia, por salir a buscar el sustento económico, alimentario, la vivienda para la familia, y además someterse a patrones abusivos por el riesgo de perder el trabajo o arriesgarse al mismo COVID-19 al estar en la primera línea de fuego por pertenecer al sector salud o a los sectores de producción que no cerraron sus puertas en el marco de la cuarentena.

Las restricciones de acceso a la información, la falta de recursos económicos, llevo a las mujeres a la dependencia económica o de cuidados, la inestabilidad en el empleo, entre otros son los impactos de la pandemia actual que pueden desencadenar en trastornos de ansiedad, depresión que agudice la violencia de género y se desencadene en una pandemia más aguda que la COVID 19 que perdure en el tiempo sin tener respuesta efectiva de los Estados. Por lo que es necesario, que se considere las recomendaciones de los organismos internacionales sobre políticas públicas encaminadas a contener y disminuir la violencia durante y pospandemia. No se conoce cuánto tiempo va a durar la pandemia por la COVID - 19; pero está claro que se ha convertido en un problema de salud, social, económico especialmente para las mujeres que están sufriendo violencia debido al confinamiento. Las precarias políticas públicas y mecanismos implementados no están dando el efecto deseado; al contrario, cada día se conoce de más casos de violencia y femicidios, y se presenta más barreras para denunciar por la inestabilidad de prestación de servicios del aparato judicial y de salud.

Además, la falta de sensibilización en la sociedad para detectar y denunciar por no verse involucrados en procesos legales, y la inoperancia de los Estados ante un problema de gran magnitud que ha sido minimizado y no tratado como debería ser, esto ha llevado a la protesta de las mujeres en todo el mundo. Desde el punto de vista de la política jurídica, la alerta que realizaron 
las organizaciones internacionales - nacionales, las organizaciones de mujeres, la sociedad civil, sobre la violencia que están sufriendo las mujeres por parte de sus parejas durante la COVID-19, son importantes porque hacen un llamado para que el Estado adopte medidas mediáticas en coordinación interinstitucional, con la finalidad de que se especialice a equipos para acudir al auxilio inmediato y se aplique racionalmente las medidas de protección para la víctima, se espera que los Estados procuren acoger y poner en marcha

En Ecuador, se incrementó la violencia de género y los femicidios durante la pandemia; sin embargo, el confinamiento, el miedo y la vergüenza les obliga a mantener el silencio y no denunciar al agresor. Por otro lado, la falta de atención personalizada de los entes judiciales, la falta de recursos tecnológicos y económicos, la falta de cifras oficiales, no permiten visibilizar el impacto y el riesgo al que están expuestas las mujeres. Sin embargo, las organizaciones civiles de mujeres estando confinadas, no han dejado de levantar la voz de protesta a través de las redes sociales alertando de los sucesos de violencia y femicidio, exigiendo al Estado crear políticas de prevención y atención con enfoque de género, se ha implementado ciertas recomendaciones a través de mandatos formales del Estado, que no son plasmados debido a falta voluntad política, de presupuesto, la falta de coordinación interinstitucional. Las organizaciones civiles son las más visibles frente a la violencia de género. Pero aún, queda pendiente por conocer:

¿Cuáles son las cifras reales de violencia de género y femicidios que causo la pandemia por COVID-19, en Ecuador?

¿Cuál es el impacto de la pandemia por COVID-19 en las mujeres rurales e indígenas y afrodescendientes (más vulnerables) del Ecuador?

\section{REFERENCIAS DE FUENTES CITADAS}

ACNUDH. (Alto Comisionado de las Naciones Unidas para los Derechos Humanos). Declaración sobre la eliminación de la violencia contra la mujer. 2000. Recuperado de: http://www.oas.org/DIL/ESP/1993Declaracion_sobre_la_eliminacion_de_la_violencia_contra_la_mujer.pdf.

ARTICULACIÓN REGIONAL FEMINISTA, Los derechos de las mujeres de la región en épocas de COVID-19. Estado de situación y recomendaciones para promover políticas con justicia de género. Buenos Aires, mayo 2020.

BARRAGAN, Almudena, Y RODRÍGUEZ, Darinka. Las llamadas por violencia de género en México aumentan $60 \%$ durante la cuarentena, (03 de 04 de 2020), EL PAÍS. Recuprado de: https://verne.elpais.com/ verne/2020/04/02/mexico/1585780887_471083.html.

BUNCH, Charlotte, HINOJOSA, Claudia y REILLY, Niamh. Los derechos de las mujeres son derechos humanos, crónica de una movilización mundial. Edamex S.A.

BBC,R.N. (Corporación Británica de Radiodifusión). Coronavirus: la preocupación por las víctimas de violencia de género que tienen que convivir en cuarentena con su agresor (y dónde buscar ayuda). (24 de marzo de 2020). Recuperado de: https://www.bbc.com/mundo/noticias-52009140. 


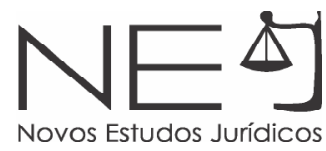

CEPAL (Comisión Económica para América Latina y el Caribe). Medidas y acciones impulsadas por los Gobiernos de América Latina y el Caribe frente al COVID-19 en áreas clave para la autonomía de las mujeres y la igualdad de género. 2021, consultado en: https://www.cepal.org/sites/default/files/events/ files/220222_documento_mapeo_medidas_covid-19_rev_dag_002.pdf

CEPAL (Comisión Económica para América Latina y el Caribe). Construir un futuro mejor: acciones para fortalecer la Agenda 2030 para el Desarrollo Sostenible (LC/FDS.4/3/Rev.1). Santiago, 2021. Consultado en: https://repositorio.cepal.org/bitstream/handle/11362/46682/6/S2100125_es.pdf.

DIARIO INTERNACIONAL, Aumentan reportes de violencia de género durante confinamiento en España, (04 de abril de 2020), Recuperado de : https://www.adn40.mx/noticia/internacional/notas/2020-04-03-14-59/ italia-protesta-contra-feminicidio-de-doctora-que-combatia-el-covid-19

EL PAÍS. Encerradas con el maltratador, (20 de marzo de 2020). Recuperado de : https://elpais.com/ sociedad/2020-03-20/encerradas-con-su-maltratador.html. México 2000.

FGE. (Fiscalía General del Estado). Informe de delitos a agosto 2020, FGE, 2020.

GALTUNG J. Violence, peace, and peace research, Journal of Peace Research, vol., 6, pp. 167-191,1969 en

GARCÍA DE BLAS, Elsa. Encerradas con su matratador, (20 de marzo de 2020), EL PAís, recuperado de: https://elpais.com/sociedad/2020-03-20/encerradas-con-su-maltratador.html.

GARCÍA, Dora. En busca de una cultura de Paz frente a la violencia: El caso de la Trata de personas. Frontera Norte, vol. 26, núm. 51. 2014, págs. 181-192.

GARCÍA, Noemi. Coronavirus y violencia de género: un binomio peligroso. Ayuda en Acción, (20 de abril de 2020). Consultado en: https://ayudaenaccion.org/ong/blog/mujer/coronavirus-violencia-genero/.

KOHAN, M., Indignación en el Ministerio de Igualdad ante el afán de Interior por minimizar la violencia machista en cuarentena. Diario Público de Madrid, 31 de marzo de 2020, Recuperado de:

https://www.publico.es/sociedad/violencia-genero-coronavirus-indignacion-ministerio-igualdad-afaninterior-minimizar-violencia-machista-cuarentena.html.

KLUGE, Hans. La OMS alertó que la violencia contra las mujeres aumento un $60 \%$ durante las cuarentenas (08 de mayo de 2020), Infobae. Recuperado de: https://www.infobae.com/america/mundo/2020/05/07/ la-oms-alerto-que-la-violencia-contra-las-mujeres-se-incremento-un-600-por-las-cuarentenas/.

LARRAÍN, S. Los orígenes y el control de la violencia doméstica en contra de la mujer, 1993, en GÓMEZ, Elsa, Género, Mujer y Salud en las Américas, Washington D.C. Organización Panamericana de la Salud,1993. págs. 202 - 212.

LORENTE, Miguel. Violencia de género en tiempos de pandemia y confinamiento. Revista española de medicina legal, 2020, Pgs. 139-145.

MOLINA, Estefanía. Factores de riesgo y consecuencias de la violencia de género en Colombia, Tempus Psicológico, 2019, Pgs. 15-36.

MAQUEDA, María Luisa. La violencia de género: Entre el concepto jurídico y realidad social, Revista Electrónica de Ciencia Penal y Criminología 2006, p.4.

MINDS, D. M., Confinamiento por coronavirus: la violencia doméstica y el abuso infantil podrían aumentar. 20 de 03 de 2020, recuprado de: w.com/es/confinamiento-por-coronavirus-la-violencia-doméstica-y-elabuso-infantil-podrían-aumentar/a-52862705.

MOLINA, Violeta. La denuncia del negocio de la violencia de género con el COVID 19, pero nada para los varones confinados con sus maltratadoras, 2020. Recuperado de: https://www.efe.com/efe/espana/ sociedad/violencia-machista-prohibido-dar-la-espalda-durante-pandemia/10004-4198583. 
MUÑOZ, Rosa. América Latina lucha contra la violencia de género en tiempos del coronavirus. DW Made For Minda , (03 de 31 de 2020). Recuperado de : https://www.dw.com/es/am\%C3\%A9rica-latina-lucha-contrala-violencia-de-g\%C3\%A9nero-en-tiempos-del-coronavirus/a-52971832.

NOTICIAS ONU, El COVID-19 golpea tres veces a las mujeres: por la salud, por la violencia doméstica y por cuidar de los otros, 2020. Recuperado de: https://news.un.org/es/story/2020/03/1471872

NACIONES UNIDAS. IV Informe de la Cuarta Conferencia Mundial sobre la Mujer, 1995, p.52.

Noticias ONU (Organización de las Naciones Unidas). Las trabajadoras domésticas en jaque por el coronavirus, (20 de junio de 2020).

OEA/CIM. (Organización de los Estados Americanos/ Comisión Interamericana de Mujeres). Covid-19 en la vida de las mujeres. Razones para reconocer los impactos diferenciados. 2020. Consultado en: https:// www.oas.org/es/cim/docs/ArgumentarioCOVID19-ES.pdf

OMS (Organización Mundial de la Salud). Obtenido de Violencia contra la Mujer, 2017, consultado en: https:// www.who.int/es/news-room/fact-sheets/detail/violence-against-women.

OMS (Organización Mundial de la Salud). La OMS caracteriza a COVID-19 como una pandemia. 2020, consultado en: https://www.paho.org/es/noticias/11-3-2020-oms-caracteriza-covid-19-como-pandemia.

Resolución 48/104 de la Asamblea General de las Naciones Unidas. Cfr. IV Conferencia Internacional de Beijing de. Revista del Ministerio de Trabajo y Asuntos Sociales. Número 42. Pág. 231. Cfr. Convención Interamericana para prevenir, punir y erradicar la violencia contra la mujer, de 1994.

SAGOT, Montserrat. The Critical Path of Women Affected by Family Violence in Latin America Case Studies From 10 Countries. Violence Against Women, 2005, pgs. 1292-1318.

ONU (Organización de las Naciones Unidas), Las trabajadoras dompesticas en jaque por el coronavirus, (20 de junio de 2020), Noticias ONU.

PALOMINO F. Encuesta violencia de género contra las mujeres durante el confinamiento por el COVID-19, 2020. Recuperado de: https://www.cepam.org.ec/wp-content/uploads/2020/09/Datos_Violencia-de-Genero_ ECU2020.pdf.

PRIMICIAS, 20.000 casos de violencia de género e intrafamiliar reportados desde marzo 2020, Recuperado de: https://www.primicias.ec/noticias/sociedad/ecuador-casos-violencia-genero-emergencia/

SENDA. Segunda encuesta online efectos del COVID-19 en el uso de alcohol y otras drogas en Chile 2021. Recuperado de: https://www.senda.gob.cl/wp-content/uploads/2021/06/Segunda-Encuesta-SENDA.pdf

RADIO VALENCIA. El confinamiento aumenta el riesgo de las mujeres maltratadas. Recuperado de : https:// cadenaser.com/emisora/2020/04/01/radio_valencia/1585745709_613957.html.

20 Minutos. Cruz roja advierte que el confinamiento puede aumentar las agressiones a las victimas de violencia de género. Google, (2 de abril de 2020). Obtenido de: https://www.20minutos.es/noticia/4214837/0/ cruz-roja-advierte-que-el-confinamiento-puede-aumentar-agresiones-a-las-victimas-de-violencia-degenero/.

Recebido em: 20/01/2021

Aprovado em: 08/03/2021

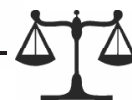

